¿Interseccionalidad en América Latina y el Caribe? La experiencia de la Red de Mujeres Afrolatinoamericanas, Afrocaribeñas y de la Diáspora desde 1992 hasta la actualidad Lucía Busquier

Con X (N. $\left.{ }^{\circ} 4\right), \mathrm{e} 023,2018$

ISSN 2469-0333 | https://doi.org/10.24215/24690333e023

http://perio.unlp.edu.ar/ojs/index.php/conequis

FPyCS | Universidad Nacional de La Plata

La Plata | Buenos Aires | Argentina

\title{
¿Interseccionalidad en América Latina y el Caribe?
}

Intersectionality in Latin America and the Caribbean?

\author{
Lucía Busquier \\ lu.busquier@gmail.com \\ https://orcid.org/0000-0003-1174-2221 \\ Facultad de Filosofía y Humanidades \\ Universidad Nacional de Córdoba \\ Argentina
}

\section{Resumen}

El objetivo principal que orienta este artículo es exponer los avances de una investigación en curso en la que se propone indagar las formas que adoptó la interseccionalidad como perspectiva teórica y política en América Latina y el Caribe desde la década del noventa hasta la actualidad. Así, a partir del estudio de la Red de Mujeres Afrolatinoamericanas, Afrocaribeñas y de la Diáspora (RMAAD), nacida en 1992 en la República Dominicana, serán abordados los primeros pasos de dicha investigación.

Palabras clave | interseccionalidad, mujeres de color, género, raza

\section{Abstract}

The main objective of this article is to expose the progress of an ongoing research where it is proposed to investigate the forms taken by intersectionality as a theoretical and political perspective in Latin America and the Caribbean from the nineties to the present. Thus, from the study of the Afro-Latin American, AfroCaribbean and Diaspora Women Network, born in 1992 in the Dominican Republic, the first steps of this research will be addressed.

Keywords | intersectionality, women of color, gender, race 


\title{
en América Latina y el Caribe?
}

La experiencia de la Red de Mujeres Afrolatinoamericanas, Afrocaribeñas y de la Diáspora, desde 1992 hasta la actualidad

\author{
Por Lucía Busquier
}

\section{Introducción}

En este artículo se busca compartir los avances de una investigación en curso en la que se analizan las formas que adoptó la interseccionalidad como perspectiva teórica y política en América Latina y el Caribe desde la década del noventa hasta la actualidad. Tomando como punto de partida el surgimiento de dicha perspectiva impulsada por el feminismo negro de los Estados Unidos en la década del setenta, se propone estudiar qué formas adoptó la interseccionalidad en el contexto latinoamericano contemplando, por un lado, las producciones teóricas que se desarrollaron durante la década del noventa en América Latina y el Caribe y, por el otro, las trayectorias militantes de las mujeres de color que impulsaron la Red de Mujeres Afrolatinoamericanas, Afrocaribeñas y de la Diáspora (RMAAD), nacida en 1992 en la República Dominicana.

En ese marco y en términos específicos, en este artículo se reconstruye, en primer lugar, el estado del arte sobre los estudios de género desde una perspectiva interseccional y los feminismos en América Latina y el Caribe. En segundo lugar, se investiga el surgimiento de la RMAAD en 1992, considerando sus debates, sus resoluciones y sus objetivos principales, en tanto se posicionó como una organización que buscaba enfrentar la discriminación racial y de género. 
La RMAAD nació en 1992 en el marco del Primer Encuentro de Mujeres Negras de América Latina y el Caribe. Entre sus principales objetivos se encontraban: luchar contra el racismo y las opresiones de género; generar una articulación internacional de mujeres afrodescendientes, latinas y caribeñas para enfrentar de manera coordinada esas opresiones en toda la región latinoamericana; e impulsar la participación de dichas mujeres en diversos espacios políticos. Precisamente, por tratarse de una organización antirracista y antisexista compuesta por mujeres de color, su estudio permitirá reflexionar sobre cómo se pensó la intersección de estas opresiones en América Latina y el Caribe, tomando como punto de partida las declaraciones y los documentos políticos publicados por la red.

En este escrito utilizamos el término «mujeres de color» adoptado en la década del setenta por las feministas negras de los Estados Unidos, quienes pretendían conformar un movimiento solidario y horizontal que permitiera alcanzar una coalición política entre todas las «minorías» raciales y étnicas (Lugones, 2008; Golubov, 2016). Si bien sabemos que existen múltiples formas de denominación y de autodenominación para las poblaciones africanas traídas como mano de obra esclava a América durante la etapa colonial, al igual que para las poblaciones originarias de América existentes previas a la conquista, y a los nuevos grupos étnicos surgidos de ese intercambio, estas designaciones fueron producidas en diversos contextos históricos y con propósitos políticos y sociales distintos. A los fines de este artículo, y teniendo en cuenta los debates actuales existentes en torno a ello, no buscaremos discernir dicha discusión sino que elegimos utilizar el término «mujeres de color» y «mujeres afrodescendientes», para seguir con la misma designación que adoptaron las activistas y las escritoras que en esta investigación estudiamos.

En relación con el marco conceptual, el presente trabajo se inscribe dentro del campo de la historia del feminismo y de los estudios de género, y retoma, para ello, una perspectiva crítica que permita problematizar una serie de categorías teóricoconceptuales emergentes del corpus documental sobre el cual se desarrollará la investigación. En continuidad con mi trabajo final de licenciatura, en el que desarrollé una investigación sobre una organización de «mujeres del Tercer Mundo» en los Estados Unidos con el objetivo de reconstruir los orígenes de la interseccionalidad, en este estudio se analiza cómo se problematizó, en términos teóricos y políticos, el entrecruzamiento de opresiones que enfrentan las mujeres de 
color en el contexto latinoamericano y caribeño desde la década del noventa hasta la actualidad. La elección de este período se debe a que a partir de dicha década comenzaron a emerger gran cantidad de producciones teóricas sobre esta perspectiva, al tiempo que también surgieron las primeras redes de articulación política a nivel regional — como fue el caso de la RMAAD - que aún perduran como forma organizativa en el campo de los movimientos políticos y sociales.

Esta investigación, además, se inscribe en una perspectiva feminista y decolonial que intenta deconstruir las ideas y los conceptos impuestos por la matriz de dominación y por la «colonialidad del saber» (Quijano, 2016) que presuponen la construcción de un conocimiento positivista, eurocéntrico, heterosexista, racista y colonial. Por el contrario, aquí se busca contribuir a las nuevas formas de conocimiento no hegemónico que en los últimos tiempos lograron un lugar importante en los espacios académicos latinoamericanos de las ciencias sociales.

Al mismo tiempo, si bien podemos encontrar gran cantidad de producciones en inglés, los escritos desde una perspectiva interseccional son todavía escasos en nuestra región. Sobre todo, aquellos que buscan recuperar las particularidades y las singularidades de las mujeres de color en América Latina y el Caribe, superando los análisis generales que entienden al sujeto mujer de manera homogénea y los que analizan las problemáticas relacionadas con el género, la raza, la clase y la colonialidad, entre otros, de manera fragmentada. Es en este punto donde nos parece fundamental el aporte que podremos realizar a los estudios de género y a los feminismos no hegemónicos en América Latina y el Caribe.

Para el desarrollo de este escrito se optó por una estrategia metodológica cualitativa con un diseño flexible (Mendizábal, 2006) centrada en un análisis documental de fuentes escritas, es decir, las declaraciones y las resoluciones de los encuentros de la RMAAD, algunas publicadas en su página web y otras suministradas por una de sus fundadoras, Ochy Curiel.

En una próxima etapa, la cual no será abordada en este artículo, se desarrollarán entrevistas en profundidad focalizadas, principalmente, en activistas $\mathrm{y}$ en escritoras latinoamericanas y caribeñas como Yuderkys Espinosa Miñoso, Ochy Curiel y Mara Viveros Vigoya quienes residen en Colombia, entre otras. Para la selección de las entrevistadas, priorizaremos a aquellas que hayan participado en la RMAAD y que desarrollen producciones teóricas, en pos de responder a uno de nuestros objetivos, aunque este no será un condicionamiento excluyente. 


\section{Hacia la construcción de un estado del arte:}

\section{las mujeres de color como objeto de estudio}

En términos generales, la interseccionalidad hace referencia a las múltiples identidades y experiencias de exclusión, de subordinación y de opresión que atraviesan los sujetos. De algún modo, la interseccionalidad vino a dar cuenta de las posiciones múltiples a la hora de pensar en las formas de dominación y de subordinación que enfrentan los sujetos y apunta a complejizar la concepción de género al concebirla como una dimensión entre otras dentro del complejo tejido de relaciones sociales y políticas (Stolcke, 2004).

Kimberlé Crenshaw $(1989,1991)$ fue una de las primeras pensadoras que consideró la interseccionalidad al realizar un análisis sobre la violencia contra las mujeres de color. A fines de la década del ochenta y comienzos de los noventa, la autora sostenía que el feminismo contemporáneo y los discursos antirracistas no contemplaban las intersecciones que se producían entre el racismo y el patriarcado. Nattie Golubov (2016) agrega que se trata de una herramienta heurística para incorporar a las mujeres de color a los análisis teóricos y al activismo político del feminismo.

Patricia Hill Collins (1998), en contraposición a los estudios que consideran al género, la raza, la clase y la nación como sistemas separados, sostiene que la interseccionalidad permite analizarlos en conjunto, indagando cómo dichos sistemas se articulan y se combinan. Deborah King (1988) recorre los primeros postulados sobre la existencia de opresiones múltiples que llevaron a la consolidación de la perspectiva interseccional, evidenciando una estrecha relación entre la teoría y la praxis política. Kathy Davis (2008) y Anna Carastathis (2014) reconstruyen los enunciados de la interseccionalidad buscando esclarecer cuáles fueron los aportes de dicha perspectiva que sustentan su vigencia en la actualidad. Por último, Bell Hooks (2017), a partir de sus experiencias individuales permeadas por las opresiones de género, de raza y de clase, ofrece un análisis político y teórico que resignifica los límites del feminismo. A esto se le sumaron otros que incluían la cuestión de la colonialidad y el eurocentrismo, aduciendo que el feminismo occidental privilegiaba solo la mirada de las mujeres del «Primer Mundo» (Oliva Portolés, 2007). 
Los estudios sobre la interseccionalidad producidos por escritoras latinoamericanas fueron, en su mayoría, desarrollados a partir de la década del noventa. Su objetivo era deconstruir y discutir los postulados del feminismo hegemónico y occidental que no resultaban suficientes para explicar las realidades de las mujeres latinoamericanas (Espinosa Miñoso, 2009). María Lugones (2008) analiza cómo se entrecruzan las opresiones de raza, clase, género y sexualidad, y con ello explicita las conexiones entre dichas opresiones. Rocío Medina Martín (2014) propone estudiar los principales aportes de los feminismos periféricos hacia el pensamiento feminista hegemónico, a partir de una genealogía del pensamiento feminista periférico. Chela Sandoval (1991), agrega que el feminismo hegemónico del siglo XX desarrolló formas de resistencia efectivas pero que representaban solo a una parte del movimiento de liberación de las mujeres de esa época.

Mara Viveros Vigoya (2009), a partir de una selección de producciones académicas recientes en América Latina y el Caribe, examina de qué manera se entienden las intersecciones entre género, sexualidad y raza. Para la autora, tanto el racismo como el sexismo utilizan el argumento de la «naturaleza» para justificar y para reproducir las relaciones de poder, establecen una relación entre lo corporal y lo social, y visualizan a las mujeres como grupo naturalmente predeterminado a la sumisión. En otro artículo, recupera los aportes fundamentales, tanto teóricos como políticos, de la interseccionalidad, atendiendo a sus orígenes y a las contribuciones del feminismo negro y latinoamericano. Allí, Viveros Vigoya (2016) sostiene que la interseccionalidad busca visibilizar las imbricaciones que existen en las relaciones de poder, algo que dentro del feminismo negro se comenzó a plantear incluso antes de denominarla «interseccionalidad». Karina Bidaseca (2012), en tanto, propone comprender el sexismo y el racismo como dos elementos unificados a partir de considerar a las «mujeres del Tercer Mundo» como el punto de intersección entre el colonialismo, el imperialismo, el nacionalismo y los fundamentalismos culturales.

Sobre investigaciones situadas en América Latina y el Caribe, contamos con el texto de Marisol Fournier-Pereira (2014), quien ofrece un análisis sobre los feminismos lésbicos en Centroamérica a partir de una perspectiva interseccional. Por su parte, Doris Lamus Canavate (2009) recupera los estudios sobre mujeres negras / afrocolombianas para contribuir al debate actual sobre el rol que ocupan dichas mujeres en los procesos organizativos identitarios, políticos y culturales. 
La autora propone un análisis más complejo que los desarrollados por el «feminismo blanco y burgués», donde se considere el entrecruzamiento entre las variables de género, raza/etnia y clase, con el fin de visibilizar otros grupos de mujeres y poder ir más allá de la vinculación como grupo únicamente por la condición de género. En otro escrito (2009b), esta misma autora crítica los postulados eurocentristas del feminismo y rescata las particularidades de los feminismos latinoamericanos, aduciendo que los feminismos occidentales tienden a universalizar y a generalizar las problemáticas de las mujeres. Por el contrario, propone reinscribir en la historia las experiencias de las mujeres excluidas por el feminismo occidental.

Finalmente, el texto de Liset Coba y de Gioconda Herrera (2013) reflexiona sobre los nuevos desafíos para el movimiento feminista latinoamericano en el marco del contexto neoliberal iniciado en la década del ochenta. El estudio realizado por Patricia Muñoz Cabrera (2011) para Central America Women's Network (CAWN) también analiza, a partir de una mirada interseccional, cómo se vincula la violencia hacia las mujeres con la pobreza en el marco del neoliberalismo en América Latina y el Caribe. En el campo de las migraciones contamos con los estudios de María José Magliano (2013, 2015) y Adriana Piscitelli (2008), quienes estudian las particularidades de las mujeres migrantes en la Argentina desde una mirada interseccional.

Otro grupo de lecturas que nutre esta investigación está dado por los escritos sobre la historia del movimiento de mujeres de color latinoamericano, los cuales serán de suma importancia a la hora de reconstruir el contexto político en el que se insertó la interseccionalidad como perspectiva teórica y política, y la RMAAD. Uno de ellos es el de Sara Beatriz Guardia (2013), quien realiza un recorrido por la historia de las mujeres en América Latina y el Caribe desde el periodo incaico hasta finales del siglo XX. Alba Carosio (2009) estudia el feminismo latinoamericano considerando el contexto de globalización y de neoliberalismo que atraviesa dicha región en las últimas décadas. Silvia Chejter (2007) reconstruye las experiencias de diversas organizaciones de mujeres latinoamericanas y recupera sus prácticas, sus definiciones, sus objetivos y los resultados que obtuvieron a partir de su militancia. Ochy Curiel (2002) propone cuál sería la mejor estrategia para erradicar las opresiones de raza, sexo, clase y heterosexualidad que enfrentan las mujeres de color en la región. 
Por último, en lo que respecta al contexto político y social en el que se desarrollaron las redes afrodescendientes en América Latina y el Caribe, incluida la RMAAD, encontramos los textos de Agustín Lao-Montes (2009), quien analiza el campo político afrodescendiente en América Latina y el Caribe, donde se ubica la emergencia de dicho movimiento, a partir de la conformación de redes regionales, nacionales e internacionales de organizaciones indígenas, afrodescendientes, étnicas, etc. Rebecca Lemos Igreja (2014) estudia el momento en el que el término «afrodescendiente» se instaló en la escena política y social de América Latina y el Caribe, a fines de la década del ochenta y cómo los estados latinoamericanos comenzaron a pensar políticas públicas de inclusión. Finalmente, María José Becerra y Diego Buffa (2012) explican cómo, a fines del siglo XX, ante el reclamo del colectivo afrodescendiente en lo que respecta a su calidad de vida el Estado impulsaba medidas inclusivas hacia estos sectores.

\section{Las mujeres de color en América Latina y el Caribe: \\ el nacimiento de la RMAAD y sus primeras definiciones políticas}

Podemos ubicar hacia fines de la década del setenta el surgimiento del movimiento de mujeres de color en América Latina y el Caribe que, al igual que el feminismo negro norteamericano, se consolidó a partir de la separación política con el feminismo hegemónico y con el movimiento antirracista. El feminismo negro norteamericano fue un movimiento político y cultural que se consolidó en la década del setenta en los Estados Unidos, aunque podemos ubicar sus antecedentes en el período de la esclavitud norteamericana.

A mediados del siglo XX, las mujeres de color comenzaron a manifestar algunas diferencias en sus ámbitos de militancia y de activismo, y a exigir que se incluyeran análisis sobre las consecuencias de las múltiples opresiones de sexo, raza, clase, etnicidad y orientación sexual que enfrentaban. De ese modo, a partir de sus experiencias de vida y de su activismo fueron construyendo y cimentando las bases de lo que luego se conocerá como la perspectiva interseccional (Roth, 2004). 
Cabe aclarar que cuando nos referimos a feminismo hegemónico, Medina Martín (2014) sostiene que este tuvo, y tiene, cierta capacidad de enunciación política y un reconocimiento científico, considerando que existe una subordinación común que atraviesa a todas las mujeres por igual, por el simple hecho de serlo, lo que genera, ante este problema, respuestas comunes para todas las mujeres. Además, se ubica en un contexto epistemológico occidental moderno que descarta y que identifica como premodernas a otras alternativas de pensamiento que no responden a los límites eurocéntricos como sucede, por ejemplo, con el feminismo negro, chicano, indígena e islámico (Medina Martín, 2014).

Sin embargo, y sin dejar de tener en cuenta algunas similitudes, el feminismo negro latinoamericano tuvo y tiene sus particularidades, sus propios reclamos y estrategias de lucha. En ese sentido, Curiel (2007) explica que el movimiento de mujeres de color en América Latina y el Caribe pasó por tres instancias. En un primer momento, buscó delimitarse de manera identitaria de otros colectivos y reafirmar su identidad de «mujeres» y de «negras», es decir, llevó adelante una política de identidad mediante el redescubrimiento de la historia, la cultura y las creencias de la herencia africana, borradas por la dominación colonial a través del mestizaje. En una segunda etapa, iniciada en la década del ochenta, comenzó a exteriorizar y a visibilizar no solo las opresiones que debían enfrentar como mujeres afrodescendientes, sino también sus posicionamientos y sus estrategias políticas para eliminarlas. En este proceso, muchas de estas organizaciones eligieron la vía parlamentaria o la participación en organismos del gobierno y en ONG. Por último, dichas organizaciones iniciaron un proceso de articulación nacional, regional y mundial, en busca de transnacionalizar su acción política al igual que lo hacían las diversas formas de opresión producto de la globalización (Curiel, 2007).

En este contexto de regionalización y de expansión de los límites locales es donde podemos ubicar el surgimiento de la RMAAD. El primer evento que dio comienzo a dicha organización fue el III Encuentro Continental de Mujeres, celebrado en Cuba en 1986, donde, por primera vez, se planteó la idea de construir una herramienta para enfrentar las opresiones de raza y de género de manera regional. Luego, en 1990, en el V Encuentro Feminista de América Latina y el Caribe, celebrado en la Argentina, esta idea comenzó a tomar formas más tangibles con la creación de un comité que pensara cómo llevar adelante dicha idea en términos concretos (Curiel, 2007). 
Al año siguiente, en 1991, se realizó en Uruguay la primera reunión de coordinación para organizar el I Encuentro de Mujeres Negras de América Latina y el Caribe, que se celebró en 1992, y en el que finalmente nació la RMAAD. La creación de la RMAAD se enmarcó en un proceso de mayor envergadura en el que otras organizaciones de mujeres de color también impulsaron la conformación de redes y colocaron en la agenda de los debates feministas la cuestión de la raza, lo que permitió que el movimiento consolidara un liderazgo colectivo y una identidad política propia (Lao-Montes, 2009).

Este primer encuentro contó con la participación de 300 mujeres de 32 países de la región; algunas estaban organizadas en agrupaciones y en movimientos sociales, otras estuvieron presentes de manera independiente. Las trayectorias de quienes asistieron eran muy variadas: algunas provenían de la militancia feminista y otras de los movimientos antirracistas. También hubo una amplia diversidad en cuanto a las identidades sexo-genéricas, culturales, religiosas e ideológicas, entre otras (Evaluación de impacto del plan de incidencia, RMAAD, 2012).

Los objetivos propuestos en ese encuentro se orientaban, entre otras cosas, a visibilizar y a denunciar los diversos tipos de violencias y de discriminaciones que enfrentaban las mujeres de color, entendiendo al racismo desde una perspectiva de género. Además, se buscaba fomentar la participación política de dichas mujeres y acompañar la lucha de las mujeres afrodescendientes en Haití y en República Dominicana. Concretamente, esos objetivos enunciaban:

1. Trabajar conjuntamente para mejorar las condiciones de vida de las mujeres negras; 2. Combatir las ideas negativas (prejuicios y estereotipos) que se vinculan sobre la mujer negra; 3. Denunciar todo tipo de discriminación contra las mujeres negras; 4. Promover la participación de las mujeres negras en los diferentes espacios políticos y de decisión; 5. Trabajar la problemática del racismo desde una perspectiva de género; 6. Promover la comunicación, el intercambio de experiencias, la solidaridad y la destreza con otras organizaciones; 7. Apoyar la lucha de las mujeres haitianas por mejores condiciones sociales y por la solución de la crisis política; 8. Apoyar la lucha de la mujer dominicana de ascendencia haitiana por mejores condiciones, sociales y económicas (Evaluación de impacto del plan de incidencia, RMAAD, 2012, p. 9). 
Entre 1992 y 1996 la red comenzó su trabajo de desarrollo y de expansión pero con algunas dificultades económicas, de idioma y de comunicación, así como también con diversos debates y diferencias políticas. A pesar de ello, la RMAAD alcanzó algunos logros, como la distribución de un boletín informativo por varios países y la visibilización de la red como una herramienta regional. Los más importantes fueron:

1. Se mantuvo comunicación con los grupos de los países a través de un boletín que se enviaba por correo postal [...] 4. Se visibilizó el espacio de articulación regional de las mujeres negras, sobre todo, al interior del movimiento feminista y de mujeres, del movimiento negro organizado y de otros movimientos sociales [...] 8. Surgió un importante número de organizaciones de mujeres negras-afrodescendientes en varios países producto de la comunicación que la RMAAD mantuvo con algunas mujeres independientes (Evaluación de impacto del plan de incidencia, RMAAD, 2012, pp. 12-13).

Luego de este periodo de desarrollo y de expansión, en el II Encuentro de Mujeres Afrocaribeñas y Afrolatinoamericanas, realizado en Costa Rica en 1996, la RMAAD discutió sobre los alcances logrados como herramienta regional y, ante un balance no tan positivo, decidió proponerse nuevos objetivos que permitieran trabajar hacia una perspectiva que articulara las opresiones de género y de raza. Se observa aquí, además, la preocupación por participar en instancias gubernamentales o estatales, con el fin de alcanzar algunos de sus objetivos propuestos:

1. Impulsar la construcción y la consolidación de un movimiento amplio de mujeres afrolatinoamericanas y afrocaribeñas que incorpore la perspectiva étnica, racial y de género. A través de la misma pretendemos propiciar y fortalecer el intercambio y la solidaridad entre las organizaciones y las mujeres de la diáspora. 2. Visibilizar la realidad socioeconómica, política y cultural en la que vivimos las mujeres negras la cual nos coloca en una situación de discriminación y de subordinación, así como a las consecuentes violaciones a nuestros derechos humanos. 3. Incidir en las instancias gubernamentales o en los Estados que tienen que ver con la elaboración y la implementación de políticas públicas, con miras a cambiar el carácter racista que muchas veces subyace detrás de estas y propugnar 
por un modelo de desarrollo que se sustente en la reafirmación, el reconocimiento y el respeto de las identidades étnicas, raciales y de género. 4. Luchar por el cumplimiento de los convenios internacionales que beneficien directamente a las mujeres negras de Latinoamérica y el Caribe (Curiel, 2007, p. 266).

Hasta aquí se evidencia cómo, en un contexto donde diversas organizaciones buscaban una mayor articulación de sus políticas y de sus acciones a nivel regional, la RMAAD intentó impulsar una herramienta que sirviera, por un lado, para establecer vínculos entre las diversas organizaciones de mujeres de color y, de esa manera, lograr una mayor visibilización del colectivo y de las problemáticas particulares que enfrentaban, y, por el otro, para generar estrategias tendientes a combatir esas opresiones de género, raza, etnia, clase, entre otras. Las estrategias fueron muy variadas, sin embargo, una de las más importantes fue la participación en instancias gubernamentales y estatales a partir de la elaboración de políticas públicas, de censos y de convenios internacionales.

Luego de esos años de desarrollo y de crecimiento, comenzó un proceso de consolidación del que surgieron algunas definiciones políticas sobre ejes específicos. Uno de ellos fue el referido a la articulación entre las opresiones de raza y de género, principalmente, donde también se intersecaban otras categorías como la de clase. En el Taller Regional realizado en Nicaragua en 2009, la RMAAD exponía: «[...] La existencia de la red es por una razón en común: nos une la identidad de ser mujeres y ser negras» (Memoria del Taller Regional, RMAAD, 2009, p. 45).

$\mathrm{Al}$ año siguiente, en 2010, la RMAAD se presentaba como

[...] un espacio de articulación para la lucha contra el racismo, el sexismo, la discriminación racial y la pobreza. Impulsamos la consolidación de un movimiento amplio de mujeres afrodescendientes, que incorpora las perspectivas étnicas, raciales y de género en su quehacer, visibilizando la realidad de la discriminación y la violencia de los derechos humanos que vivimos, en el ámbito socioeconómico, político y cultural (Declaración de la RMAAD, 2010, en línea). 
De este fragmento se desprende que para la RMAAD era fundamental la construcción de una herramienta que permitiera enfrentar dichas opresiones de manera articulada y regionalmente. Además, señalaba que en el contexto latinoamericano, las personas afrodescendientes en general, pero las mujeres en particular, se desempeñaban como mano de obra barata, a partir de criterios de discriminación de género, de clase y de raza. Así lo explicaban en una de sus declaraciones:

[...] el sistema capitalista ha incorporado a las afrodescendientes como mano de obra, con poco valor, al mercado laboral y a la producción, basándose en la división sexual del trabajo, en los estereotipos de género, en la subordinación femenina y en las desigualdades raciales y de clase [...]. La discriminación étnico-racial y de género es constitutiva de la pobreza, de la exclusión y de la perpetuación de las desigualdades sociales históricas (Declaración de la RMAAD, 2010, en línea).

Además, para la red, el trabajo doméstico remunerado, que mayormente era ocupado por mujeres de color debido a ser consideradas de "segunda categoría», evidenciaba cómo se articulaban las opresiones de raza y de género: «Es un empleo en el que la mayor parte son mujeres afrodescendientes e indígenas que son consideradas como mujeres de segunda categoría, aun cuando tienen una legislación» (Memoria del Taller Regional, RMAAD, 2009, p. 10). En otra declaración se agregaba: «[...] muchas mujeres afrodescendientes, a base de estereotipos, se emplean en el trabajo doméstico, con las peores condiciones» (Declaración de la RMAAD, 2010, p. 3). Ante este esta realidad, la RMAAD no solo buscaba denunciar y visibilizar dicha situación, sino que también exigía al Estado algunos requisitos necesarios para mejorar la situación laboral de dichas trabajadoras:

[...] adecuación salarial de la fuerza laboral femenina, sin discriminación alguna, lo que permitirá reducir las brechas basadas en etnia y en género [...]. Que los Estados implementen un sistema de seguridad social equitativo y sin discriminación étnico-racial y de género, que garantice los derechos de las trabajadoras afrodescendientes, y aseguren que los/as empleadores/as cumplan con sus obligaciones, en el marco de las normativas laborales establecidas (Declaración de la RMAAD, 2010, en línea). 
Otro punto importante que le preocupaba a la red era el relacionado con la salud sexual y con los derechos reproductivos de las mujeres de color. Al respecto, se exigía el respeto y el cumplimiento de los derechos sexuales y reproductivos de uno de los grupos que se consideraba más vulnerado:

[...] Demandamos fortalecer la vigencia y el respeto de los derechos sexuales y los derechos reproductivos de todas las personas, sin discriminación de ningún tipo. Demandamos, particularmente, asegurar a las mujeres afrodescendientes el acceso universal a los servicios de salud sexual y de salud reproductiva integral, así como a la más amplia variedad de métodos anticonceptivos, incluido el acceso a la prevención, el diagnóstico y el tratamiento para el VIH/SIDA (Declaración de la RMAAD, 2010, en línea).

Por último, otra de las definiciones políticas más importantes que encontramos en sus documentos y en sus declaraciones es la que refiere a la articulación con otras organizaciones y movimientos sociales, algo fundamental a la hora de entender a la RMAAD como una herramienta regional pero, también, como forma de enfrentar las diversas opresiones que atravesaban a las mujeres de color y a otros grupos oprimidos. Dentro de los objetivos planteados en la Evaluación de impacto del plan de incidencia (2012), la RMAAD exponía: «1. Generar diálogos para encontrar puntos comunes con el Movimiento de mujeres / feministas, indígenas, campesinas, trabajadoras domésticas, migrantes, de Derechos Humanos y LGBTI, entre otros movimientos. 2. Generar espacios de incidencia con redes nacionales e internacionales» (p. 41).

Los fragmentos citados muestran cómo la RMAAD entendía la articulación entre las diversas opresiones que enfrentaban las mujeres de color en la región latinoamericana y caribeña, ya sea desde su lugar de trabajadoras domésticas, o en lo que respecta a su salud sexual y reproductiva. Problemáticas que, a través de una herramienta de articulación a nivel regional, la RMAAD buscaba contrarrestar. $\mathrm{Al}$ mismo tiempo, la organización también proponía implementar medidas estatales y gubernamentales que sirvieran para enfrentar dichas opresiones. 


\section{Reflexiones finales}

A partir del análisis realizado observamos, en primer lugar, cómo el activismo de la RMAAD reflejaba que su militancia se articuló a partir de comprender al sujeto mujer como un sujeto racializado atravesado por otras opresiones vinculadas a su condición de clase, etnia, nacionalidad y sexualidad, entre otras, y de aportar nuevas miradas a los postulados del feminismo tradicional heredado del pensamiento occidental y colonial. Además, eran las mujeres afrodescendientes quienes ocupaban en su mayoría el trabajo doméstico remunerado que, según la perspectiva de la RMAAD, evidenciaba la múltiple discriminación que enfrentaban dichas mujeres al ser consideradas trabajadoras de "segunda categoría", frente a lo que proponían como solución una adecuación salarial y otras medidas estatales para garantizar una igualdad en sus derechos laborales.

Segundo, la RMAAD exigía una mejor educación sexual para las mujeres de color y el cumplimiento de los derechos sexuales y reproductivos. A nuestro entender, este punto adquiere una importante relevancia si se considera la histórica violación de dichos derechos por parte del Estado, principalmente el norteamericano, sobre las mujeres de color a partir de la aplicación sistemática de políticas de control poblacional selectivo durante la primera mitad del siglo XX, como fue el caso de las esterilizaciones forzadas.

En tercer lugar, sostenemos que este reconocimiento de la multiplicidad de posiciones y de opresiones favoreció que la RMAAD articulara su activismo político con otros sectores y movimientos sociales que no eran exclusivamente de mujeres, como el movimiento afrodescendiente, el indígena o el LGBT y, al mismo tiempo, generó que estos sectores incluyan una perspectiva de género en sus acciones y en sus definiciones políticas; sobre todo, el movimiento afrodescendiente y el indígena.

Por último, suponemos que en América Latina y el Caribe una de las formas que adquirió la interseccionalidad como propuesta teórica y política fue la preocupación por diferenciarse de los postulados teóricos de carácter occidental, eurocéntrico y colonial, y contemplar las especificidades de la región latinoamericana y caribeña entendida como un espacio colonizado, no solo en términos políticos y económicos, sino también teóricos e ideológicos, lo que también se expresó como "pensamiento decolonial» o como «giro epistémico decolonial». 
De esta manera, y para concluir, lo antedicho genera una serie de interrogantes que orientarán el futuro de la investigación en curso y que servirán como guía para su desarrollo: ¿Cuál era la situación particular en la que se encontraba el feminismo latinoamericano en esos años y dónde pueden encontrarse los orígenes de este movimiento? ¿Cuál fue su posición ante la situación política y económica de América Latina y el Caribe en esas décadas? ¿Qué análisis desarrolló la RMAAD sobre las problemáticas que incidían en las realidades de las mujeres afrodescendientes en América Latina y el Caribe? ¿En qué situación se encuentra actualmente la RMAAD?

\section{Referencias}

Bidaseca, K. (2012). «Voces y luchas contemporáneas del feminismo negro. Corpolíticas de la violencia sexual racializada». En Afrodescendencia. Aproximaciones contemporáneas de América latina y el Caribe (pp. 1-11).Ciudad de México, México: Organización de las Naciones Unidas.

Buffa, D. y Becerra, M. J. (2012). «La población afrodescendiente en América Latina y el Caribe. Estado, sociedad civil y Derechos Humanos». En D. Buffa, M. J. Becerra, H. Noufouri y M. Ayala (Comps.), Las poblaciones afrodescendientes en América Latina y el Caribe. Pasado, presente y perspectivas desde el siglo XXI (pp. 333-353). Córdoba, Argentina: Instituto y Maestría en Diversidad Cultural de la UNTRF, Programa de Estudios Africanos del CEA-UNC, Programa de Relaciones Internacionales y Estudios Africanos del CIECS (CONICET-UNC) y Cátedra UNESCO sobre Diversidad Cultural (Argentina).

Carastathis, A. (2014). The Concept of Intersectionality in Feminist Theory. Philosophy Compass, 9(5), 304-314.

https://doi.org/10.1111/phc3.12129 
Carosio, A. (2009). «Feminismo latinoamericano: imperativo ético para la emancipación». En Género y globalización (pp. 229-252). Buenos Aires: CLACSO. Recuperado de http://biblioteca.clacso.edu.ar/clacso/gt/20140611041611/11caro.pdf

Muñoz Cabrera, P. (2011). Violencias interseccionales. Debates feministas y marcos teóricos en el tema de pobreza y violencia contra las mujeres en Latinoamérica, Inglaterra y Honduras (trad. Ana María Sosa Ferrari). Tegucigalpa, Honduras: Central America Women's Network (CAWN). Recuperado de https://www.oiemiseal.ifch.unicamp.br/pf-oiemiseal/public-

files/violencias_interseccionales.pdf

Chejter, S. (2007). Feminismos latinoamericanos. Tensiones, cambios y rupturas. Madrid, España: Asociación para la cooperación con el sur (ACSUR).

Coba, L. y Herrera, G. (2013). Nuevas voces feministas en América Latina: ¿continuidades, rupturas, resistencias? Presentación de Dossier. Íconos. Revista de Ciencias Sociales, (45), 17-23. https://doi.org/10.17141/iconos.45.2013.3103

Crenshaw, K. (1989). Demarginalizing the Intersection of Race and Sex. A Black Feminist Critique of Antidiscrimination Doctrine, Feminist Theory, and Antiracist Politics. University of Chicago Legal Forum, article 8, 139-167. Recuperado de https://chicagounbound.uchicago.edu/uclf/vol1989/iss1/8

Crenshaw, K. (1991). Mapping the Margins. Intersectionality, Identity Politics and Violence against Women of Color. Stanford Law Review, 43(6), 1241-1297. Recuperado de http://ec.msvu.ca:8080/xmlui/bitstream/handle/10587/942/Crenshaw_ article.pdf?sequence $=1$ 
Curiel, O. (2002). Identidades esencialistas o construcción de identidades políticas: el dilema de las feministas negras. Otras Miradas, 2(2), 96-113. Recuperado de http://www.redalyc.org/articulo.oa?id=18320204

Curiel, O. (2007). «La Red de Mujeres Afrolatinoamericanas y Afrocaribeñas: un intento de acción política transnacional atacado por la institucionalización». En N. Francis Pisani, A. Tickner y N. Barnes, N. (Eds.), Redes transnacionales en la Cuenca de los Huracanes. Un aporte a los estudios interamericanos (pp. 253-276). Ciudad de México, México: ITAM-Miguel Ángel Porrúa.

Davis, K. (2008). Intersectionality as Buzzword. A Sociology of Science Perspective on What Makes a Feminist Theory Successful. Feminist Theory, (9), 67-85.

Espinosa Miñoso, Y. (2009). Etnocentrismo y colonialidad en los feminismos latinoamericanos. Complicidades y consolidación de las hegemonías feministas en el espacio transnacional. Feminismo latinoamericano. Revista Venezolana de Estudios de la Mujer, 14(33), 1-21. Recuperado de http://saber.ucv.ve/ojs/index.php/rev_vem/article/view/2064/0

Fournier-Pereira, M. (2014). Feminismos e interseccionalidad: aportes para pensar los feminismos lésbicos centroamericanos. Cuadernos Inter-c-a-mbio sobre Centroamérica y el Caribe, 11(2), 67- 87. https://doi.org/10.15517/c.a..v11i2.16637

Golubov, N. (2016). «Interseccionalidad». En H. Moreno y E. Alcántara (Coords.), Conceptos clave en los estudios de género (pp. 197-213). Ciudad de México, México: Universidad Nacional Autónoma de México. 
Guardia, S. B. (Ed.). (2013). Historia de las mujeres en América

Latina. Murcia, España: Centro de Estudios de la Mujer

en la Historia de América Latina (CEMHAL). Recuperado de

https://www.um.es/estructura/unidades/u-

igualdad/intranet/docs/historia-de-las-mujeres-en-america-latina.pdf

Hill Collins, P. (1998). It's all in the family. Intersections of Gender,

Race and Nation. Hypatia, 13(3), 62-82.

Hooks, B. [2000] (2017). El feminismo es para todo el mundo.

Madrid, España: Traficantes de sueños.

King, D. (1988). Multiple Jeopardy, Multiple Consciousness.

The Context of a Black Feminist Ideology. Signs, 14(1), 42-72.

Lao-Montes, A. (2009). Cartografías del campo político

afrodescendiente en América Latina. Universitas humanística, (68),

207-245. Recuperado de

http://revistas.javeriana.edu.co/index.php/univhumanistica/article/vie $\mathrm{w} / 2273$

Lemos Igreja, R. (2014). Afrodescendientes na América Latina

e Caribe: novos caminhos, novas perspectivas em um contexto global multicultural. Revista de Estudos y Pesquisas sobre as Américas, 8(1), 13-28. Recuperado de

http://periodicos.unb.br/index.php/repam/article/view/11502

Lamus Canavate, D. (2009). Mujeres negras/afrocolombianas en los procesos organizativos en Colombia: un aporte al estado del debate. Reflexión Política, 11(21), 108-125. Recuperado de https://revistas.unab.edu.co/index.php/reflexion/article/view/494/481

Lugones, M. (2008). Colonialidad y género. Tabula rasa, (9), 73-102. Recuperado de http://www.scielo.org.co/scielo.php?pid=S1794$24892008000200006 \&$ script=sci_abstract\&tlng=es 
Magliano, M. J. (2013). Los significados de vivir múltiples presencias: Mujeres bolivianas en Argentina. Migraciones internacionales, 7(1), 165-195. Recuperado de http://www.scielo.org.mx/scielo.php?script=sci_arttext\&pid=S166589062013000100006

Magliano, M. J. (2015). Interseccionalidad y migraciones: potencialidades y desafíos. Revista Estudos Feministas, 23(3), 691-712. https://dx.doi.org/10.1590/0104-026X2015v23n3p691

Medina Martín, R. (2014). Resignificaciones conceptuales y epistemológicas en el pensamiento político feminista eurocéntrico desde los feminismos periféricos. Cuadernos Electrónicos de Filosofía del Derecho, (29), 72-98. Recuperado de https://ojs.uv.es/index.php/CEFD/article/view/3247

Mendizábal, N. (2006). «Los componentes del diseño flexible en la investigación cualitativa». En I. Vasilachis de Gialdino (Coord.), Estrategias de investigación cualitativa (pp. 65-105). Barcelona, España: Gedisa.

Oliva Portolés, A. (2007). «Debates sobre el género». En C. Amorós y A. De Miguel (Eds.), Teoría feminista: de la ilustración a la globalización. De los debates sobre el género al multiculturalismo (pp. 15-60). Madrid, España: Minerva.

Piscitelli, A. (2008). Interseccionalidades, categorias de articulação e experiências de migrantes brasileiras. Sociedade e Cultura, 11(2), 263-274. https://doi.org/10.5216/sec.v11i2.5247

Quijano, A. (2016). «Colonialidad del poder, eurocentrismo y América Latina». En E. Lander (Comp.), La colonialidad del saber: eurocentrismo y ciencias sociales. Perspectivas latinoamericanas (pp. 219-264). Ciudad Autónoma de Buenos Aires, Argentina: Ciccus. 
Roth, B. (2004). Separate Roads to Feminism. Black, Chicana, and White Feminist Movements in America's Second Wave.

Cambridge, Estados Unidos: Cambridge University Press.

Sandoval, Ch. (1991). U.S. Third World Feminism. The Theory and Method of Oppositional Consciousness in the Postmodern World. Gender, (10), 1-24.

Stolcke, V. (2004). La mujer es puro cuento: la cultura del género. Revista Estudos Feministas, 12(2), 77-105.

http://dx.doi.org/10.1590/S0104-026X2004000200005

Viveros Vigoya, M. (2009). La sexualización de la raza y la racialización de la sexualidad en el contexto latinoamericano actual. Revista Latinoamericana de Estudios de Familia, (1), 63-81. Recuperado de http://200.21.104.25/revlatinofamilia/downloads/Rlef1_4.pdf

RMAAD (2009). Memoria del Taller Regional de la RMAAD. Managua, Nicaragua.

RMAAD (2010) Declaración de la RMAAD. Brasilia, Brasil. Recuperado dehttp://www.mujeresafro.org/rmaad/

RMAAD (septiembre de 2012). Evaluación de impacto del plan de incidencia política de la Red de Mujeres Afrolatinoamericanas, Afrocaribeñas y de la Diáspora RMAAD, en el período 2006-2011. Managua, Nicaragua. Recuperado de https://mipersonalisima.jimdo.com/app/download/5482025669/Evalua cionPequena + de + red + de + mujeres + en+incidencia $\% 5 B 1 \% 5 D . p d f ? t=14$ 97471635 